\title{
The difference of intravascular injection rate during cervical transforaminal epidural block using blunt needle compared to sharp needle.
}

Saeyoung Kim, Seungyeon Chung, Hyunho Shin, Suyoung Moon, Jihyo Kim, Chanhee Jee

Department of Anesthesiology and Pain Medicine, School of Medicine, Kyungpook National University, Daegu, Republic of Korea

\section{Introduction}

Transforaminal epidural block (TFEB) with local anesthetics and steroid is an effective treatment option of spinal radicular pain. However, during TFEB intravascular injection should be avoided, which can cause fatal neurologic deficits such as spinal infarction and cerebral infarction.

To reduce complications of intravascular injection, several methods have been proposed such as using short-beveled or blunt-type needles, and using large-diameter needles, using non-particulate steroids, and using imaging modality such as real-time fluoroscopy (RTF) or digital subtraction angiography (DSA).

Blunt needles are considered to displace instead of penetrate vessels due to their dull needle tip. Therefore, to avoid intravascular injection of steroids during TEB, the use of blunt needles has been suggested. Animal studies have shown a reduction of incidence of arterial puncture and bleeding with the use of blunt compared to sharp needles. During lumbar TEB, several studies have found that the use of blunt needles could reduce intravascular injection and paresthesia compared to sharp needles.

\section{Methods}

After IRB approval, We prospectively examined 108 cervical TFEBs. Inclusion criteria of this study were patients over 18 years of age, patients with radiating pain from spinal stenosis and herniated nucleus pulposus. Exclusion criteria were pregnancy, allergic to contrast media, patient refusal, and patients with persistent contraindication to nerve block such as coagulopathy and infection of the injection site.

Under fluoroscopic guidance, CTEBs were performed using a $22 \mathrm{G}$, 8.9-cm, sharp needle (Hakko Co., Chikuma-shi, Nagano-gen, Japan) or a 22G, 7.6-cm, COUDE blunt nerve block needle (Epimed, Marlbourogh, England). After confirmation of final needle positioning using biplanar fluoroscopy, $2 \mathrm{~mL}$ of nonionic contrast medium (Omnipaque 300, GE Healthcare, Little Chalfont, Buckinghamshire, UK) was injected at the rate of $0.5 \mathrm{~mL} / \mathrm{s}$ with real time fluoroscopy (RTF). Intravascular injection was defined as the contrast medium spreading out through the vascular channel. If intravascular injection occurred, the needle positioning was changed. The procedure time was measured from insertion of the needle to end of administration of the contrast medium to confirm successful CTEB.

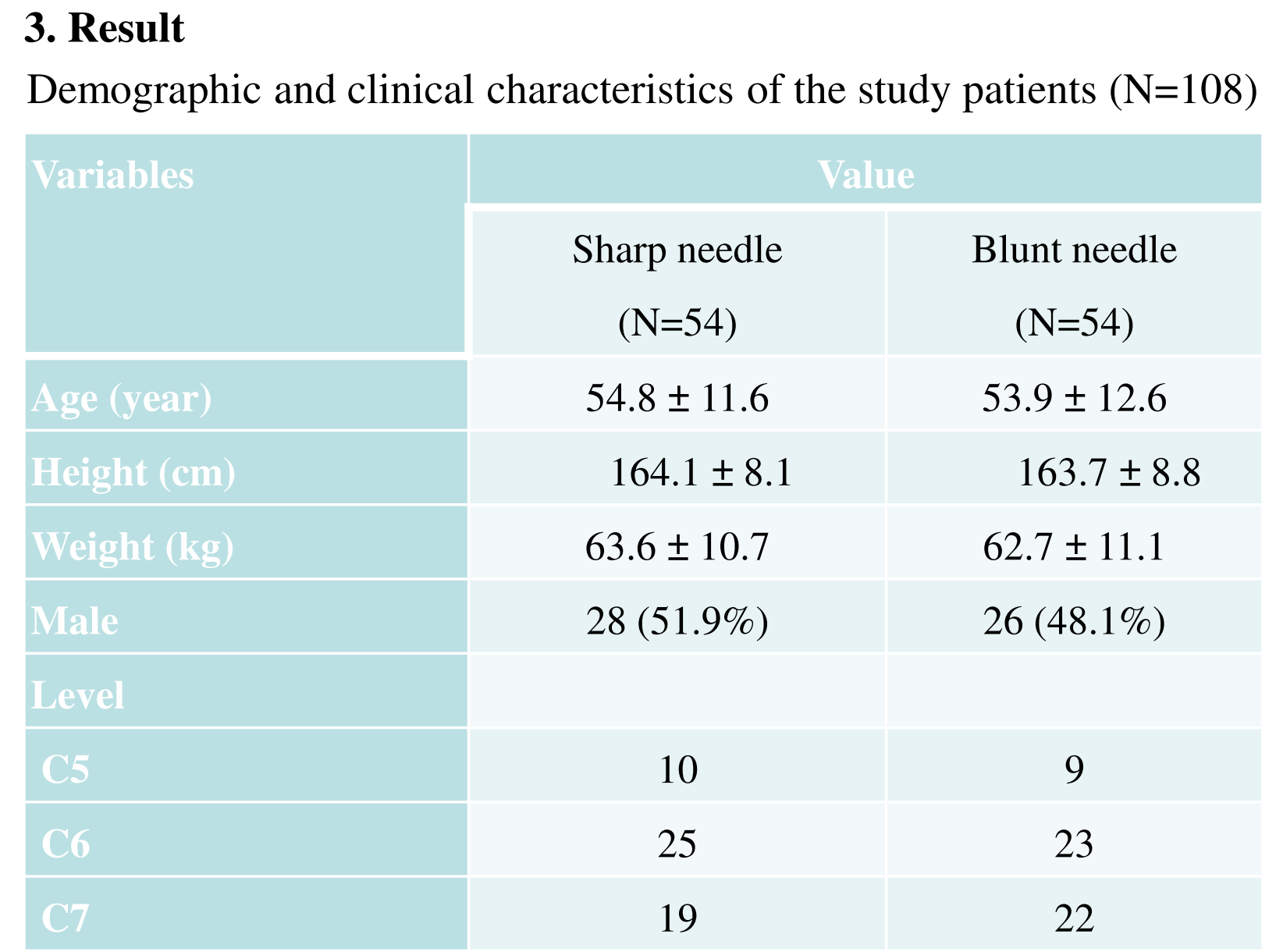

Incidence of intravascular injection during cervical transforaminal epidural block by level.

\begin{tabular}{|l|c|c|c|c|}
\hline & \multicolumn{2}{|c|}{ Sharp needle } & \multicolumn{2}{|c|}{ Blunt needle } \\
\hline injections & $\begin{array}{c}\text { Number of IV } \\
\text { injection (\%) }\end{array}$ & $\begin{array}{c}\text { Number of } \\
\text { injections }\end{array}$ & $\begin{array}{c}\text { Number of IV } \\
\text { injection (\%) }\end{array}$ \\
\hline C5 & 10 & $3(30.0 \%)$ & 9 & $3(33.3 \%)$ \\
\hline C6 & 25 & $10(40.0 \%)$ & 23 & $8(34.8 \%)$ \\
\hline C7 & 19 & $6(31.6 \%)$ & 22 & $7(31.8 \%)$ \\
\hline Total & 54 & $19(35.2 \%)$ & 54 & $18(33.3 \%)$ \\
\hline
\end{tabular}

There was no significant difference in the rate of intravascular injection between the blunt-needle and the sharp-needle groups (35.2\% vs $33.3 \%$, $\mathrm{P}>0.05)$.

The procedure time was longer in the blunt-needle group than in the sharp-needle group $(101 \pm 12.4 \mathrm{~s}$ vs $56.67 \pm 8.3 \mathrm{~s}, \mathrm{P}<0.001)$. There were no serious complications such as spinal cord or cerebral infarction and infection.

\section{Discussion}

Smuck et al. reported that there were no differences in intravascular injection rate among Chiba, Quincke, pencil-point, and blunt tip needles during lumbosacral TEB.

In the present study, the blunt needle did not reduce intravascular injection compared to the sharp needle during CTEB. In addition, procedure time in the blunt-needle group was longer than in the sharpneedle group.

\section{Conclusion}

Based on our findings, the blunt needle was not proven safer than the sharp needle for decreasing the rate of intravascular injection during CTEB. In addition, the use of the blunt needle increased procedure time compared to the sharp needle. 